\title{
FECUNDACIÓN ASISTIDA Y CONSENTIMIENTO INFORMADO: ¿QUÉ TAN INFORMADO ES DICHO CONSENTIMIENTO?
}

Ronald Cárdenas Krenz*

Recibido: 01.10.2015

Aprobado: 15.10 .2015

RESUMEN

El presente artículo trata acerca de los riesgos para la salud que resultan de la aplicación de la fecundación in vitro, tema poco difundido en el medio, no obstante su especial importancia y su necesidad para hablar de un verdadero consentimiento informado.

\section{ABSTRACT}

This article is about the risks for health that could be caused by the in vitro fertilization, a topic that it is not much spread in our society, nevertheless it is a matter of special importance to talk about a real informed assent.

\section{PALABRAS CLAVE}

Fecundación in vitro. Fecundación asistida. Riesgos.

\section{KEY WORDS}

In vitro fertilization. Assisted fertilization. Risks of the FIV.

\section{INTRODUCCIÓN}

Con más de 5 millones de nacimientos desde 1978, no cabe duda de que la fecundación asistida constituye una prác- tica muy difundida. Tan solo en Estados Unidos, en el año 2012, de acuerdo a la Sociedad de Tecnologías de Reproducción Asistida, se realizaron 165.172 procedimientos de fertilización in vitro, superando en 2.000 el número de bebés probeta registrado en el $2011 ;^{1}$ y se calcula que la industria de la reproducción asistida norteamericana gene-

* Past Decano y profesor de la Facultad de Derecho de la Universidad Femenina del Sagrado Corazón. Miembro del Instituto de Investigación Científica de la Universidad de Lima y profesor de la Facultad de Derecho. Miembro Correspondiente de la Academia de Derecho y Ciencias Sociales de Córdoba (Argentina), Profesor en la Universidad ESAN, en la Maestría de la Universidad San Martín de Porres y en la Maestría de la USAT. Ha sido miembro del Comité de Ética de la Facultad de Biología de la Universidad Nacional Mayor de San Marcos. Correo electrónico: ronald@unife.edu.pe

1 En cuanto a algunos riesgos vinculados con las técnicas de reproducción asistida, observa la Academia Pontificia para la Vida, en su Comunicado Final de la X Asamblea General (21.02.2004) que, aparte de su cada vez más frecuente uso como un apresurado recurso como única forma de tratamiento útil, "... se vislumbra en el horizonte un fenómeno todavía más inquietante: (...) la instalación progresiva de una nueva mentalidad, según la cual el recurso a las ARTs, (técnicas de reproducción asistida) podría representar, con respecto a la vía natural, el proceder directo y preferencia de traer al mundo un hijo, pues por medio de estas técnicas es posible ejercer un control más eficaz de la calidad del concebido para ajustarla a los deseos de quien la encarga".

Todo ello contribuye a considerar al hijo nacido mediante las ARTs como si fuera un producto, cuyo valor depende de su buena calidad, sometida a exigentes controles y cuidadosamente seleccionada. La consecuencia dramática de esta nueva 
ra ganancias por 3.300 millones de dólares al año. ${ }^{2}$

Incluso, en Costa Rica, a fines del 2012, habiendo estado prohibida la fecundación asistida, un fallo de la Corte Interamericana de Derechos Humanos, ordenó a dicho país levantar el impedimento. ${ }^{3} \mathrm{Si}$ bien el fallo es discutible ${ }^{4}$, lo cierto es que dicha resolución ha sido utilizada para reforzar la recurrencia a la referida técnica.

Empero, ¿es inocua su aplicación?, ¿conocen sus usuarios todos los riesgos que puede implicar?, ¿qué tan informado es el consentimiento que dan las personas que se someten a dicha técnica? 0 , mejor dicho, ¿qué tan informadas están las personas que brindan su consentimiento al uso de la fecundación asistida?

Al analizar la cuestión, debemos empezar por tener presente que el solo deseo de hacer algo y la posibilidad de lograrlo, no bastan para justificar cualquier práctica. No todo lo que se puede hacer, se debe hacer.

Las técnicas de reproducción asistida aparecieron con el objeto de ayudar a las personas con problemas para concebir. Empero, hoy se vienen aplicando sin reparar demasiado en su finalidad original, pareciendo, a veces, haberse dejado más bien para el capricho de unos y el negocio de otros.

Es así que, según datos de Michael Saendel, citados por Rincón ${ }^{5}$, dado que en Estados Unidos las gestantes por maternidad sustituta pueden cobrar entre U.S. $\$ 20.000$ y 25.000 por embarazo, muchas parejas recurren a países como la India, en donde el costo del "servicio" es de entre US \$ 4.500 y 7.500; de esta manera, la maternidad sustituta es hoy en día un mercado que crece cada día más a nivel internacional, constituyendo una triste forma de "turismo reproductivo"6 (Rincón Castellanos, 2012, pág. 101).
Según un informe del Centro para la Investigación Social de la India, el turismo reproductivo mueve alrededor de 332 millones de euros al año, existiendo cerca de 600 clínicas de procreación asistida. Recientemente, el gobierno indio acaba de aprobar la posibilidad de importar embriones congelados, lo que seguramente favorecerá aún más este lucrativo negocio. ${ }^{7}$

Tailandia, por otro lado, se había convertido, al igual que la India, en un próspero territorio para el turismo reproductivo. Sin embargo, de acuerdo a lo dispuesto por el nuevo gobierno, en agosto del 2014, ya no se permitirá la maternidad con fines comerciales (la que se penaría con diez años de cárcel y multa), restringiéndose la subrogación a los parientes.

Un factor que ha contribuido con el protagonismo de la técnica y una visión más complaciente, a pesar de implicar un tratamiento muy

actitud es la eliminación sistemática de aquellos embriones humanos que resultan carentes de la calidad considerada suficiente de acuerdo con parámetros y criterios inevitablemente cuestionables” (Sgreccia, 2008, pág. 17).

2 Fuente: http://argentinosalerta.org/print/2623. (Consultada el 18.10.2013)

3 Caso Atavia Murillo y otros vs. Costa Rica, sentencia de 28 de noviembre de 2012: “2. El Estado debe adoptar, con la mayor celeridad posible, las medidas apropiadas para que quede sin efecto la prohibición de practicar la FIV y para que las personas que deseen hacer uso de dicha técnica de reproducción asistida puedan hacerlo sin encontrar impedimentos al ejercicio de los derechos que fueron encontrados vulnerados en la presente Sentencia (...)".

4 Véase la Declaración de Guanajuato, de fecha 20 de abril de 2013, sobre la Fecundación in Vitro: http:// declaraciondeguanajuato.org

5 Agrega Rincón además que: “La maternidad sustitutiva es una práctica que se consolida día a día como mercado, se rige por las reglas de la oferta más económica y encuentra en las mujeres con escasos recursos y con hijos, las candidatas más adecuadas para obtener el mismo objetivo, pero a más bajo costo” (Rincón Castellanos, 2012, pág. 102).

6 Señala Sambrizzi criticando este tipo de acuerdos: "el objeto del contrato consiste en un ser humano, permitiendo un acuerdo de esa especie que las mujeres pobres que necesitan desesperadamente un ingreso extra y que se prestan a un acto que no puede sino ser calificado de inmoral, sean explotadas por aquellas personas (solas o en pareja, varones o mujeres) con bienes suficientes, que quieren tener un hijo, pero que no pueden o no quieren pasar por las incomodidades del embarazo" (Sambrizzi, 2012). "La llamada subrogación de útero o madre de alquiler es un ejemplo de cómo se puede obligar a un embrión a desarrollarse en un medio que le es ajeno, cercenando su derecho a desarrollarse en el seno de la madre que la ha engendrado" (Vila-Coro, Introducción a la Biojurídica, 1995, pág. 209).

7 Fuente: British Medical Journal 2014; 348: f403, tomado de:

http: / / www.observatoriobioetica.org/2014/03/el-mercado-reproductivo-en-india/?utm_source=wysija\&utm_ medium=email\&utm_campaign=432. (Consultada el 20.08.2014). 
costoso -como señalan López y Aparisi- ha sido la publicidad que le han hecho el que recurran a ella actores y cantantes famosos como Sarah Jéssica Parker, Elton John, Nicole Kidman, Miguel Bosé o Ricky Martin. Todo ello sin contar con una vasta publicidad, ya sea directa o indirecta, a través de los medios de comunicación, el cine, las telenovelas, etc. (López Guzmán, José y Aparisi, Ángela, 2012, pág. 257).

La cuestión se hace más relevante si tenemos en cuenta la opinión de Freitas Drumond, cuando afirma que:

"La última batalla en pro de la dignidad humana se pelea diariamente en los laboratorios de genética molecular y en las clínicas de reproducción asistida, donde se manipula el ADN humano y donde embriones son seleccionados conforme sus características genéricas y/o aptitudes seleccionadas por sus progenitores" (De Freitas Drumond, 2012, pág. 67). ${ }^{8}$

\section{UNA POLÉMICA CASUÍSTICA}

Si bien, en torno a la fecundación asistida, hay muchas historias de felicidad; también hay toda una polémica en la casuística vinculada con el tema, como lo sucedido en el año 2008, en Inglaterra, cuando una pareja de sordos: Tomato Lichy y Paula Garfields, apelando a la manipulación genética, exigía que los médicos les ayudaran a concebir un niño creado ex profesamente sordo, seleccionándolo genéticamente. ${ }^{9}$

Un caso similar ocurrió en Estados Unidos, en donde una pareja de lesbianas sordomudas, residentes en Washington: Sharon Duchesneau y Candy McCullough, gracias a un tratamiento de fertilización in vitro lograron tener un hijo sordo, para cuyo fin buscaron a un amigo sordo congénito a efectos de asegurarse que el niño nazca también sordo. La pareja había tenido antes otra hija concebida premeditadamente sordomuda. ${ }^{10}$

Otro caso que pone en evidencia la superficialidad en la que puede derivar el tema, es el de un aviso publicado en el New York Times, en su sección Money and Business, en agosto de 1996, que decía, a propósito del "servicio" de reproducción asistida, al más puro estilo comercial: "It's a Baby or it's your money back" (Vila-Coro, 1997, pág. 23). ${ }^{11}$

También puede citarse aquí una oferta del Centro de Medicina Reproductiva en Albury (Sidney), que puso un anuncio en un periódico estudiantil de Canadá ofreciendo dos semanas de vacaciones gratis en Australia a los muchachos que quieran donar su esperma. La oferta comprendía US $\$ 5.180$, que incluían pasajes, alojamiento y gastos de dos semanas.

A inicios de agosto de 2004, una noticia volvió a poner en entredicho la aplicación de la fecundación in vitro, a propósito de un caso de vientre de alquiler: un matrimonio australiano que alquiló un vientre a una mujer en Tailandia, decidió abandonar a uno de los bebés, nacidos en diciembre de 2013, porque tenía sín-

8 De Freitas Drumond, José Geraldo (2012). “Ética, bioética y los desafíos del siglo XXI”. En: Derecho No. 69, revista de la Facultad de Derecho de la Pontificia Universidad Católica del Perú. Lima: PUCP, pag. 67.

9 La pareja argumentaba que su sordera no era una discapacidad sino una cualidad, siendo parte de una comunidad diferente, de una minoría lingüística de la que deseaban también sean parte sus hijos (Diario El País. Edición Digital. Madrid: 11.03, 2008).

$10 \mathrm{http://www.bioeticaweb.com/content/view/4049/781/.} \mathrm{(Consultada} \mathrm{el} \mathrm{20.01.2014).}$

11 Como señala Serrano, la procreación asistida tiende a generar paradojas: la justicia exige el consentimiento de ambos padres para implantar un embrión congelado pero, a la vez, acepta que nazcan niños sin padre legal, como en la maternidad mediante semen de donante; el problema -sostiene- "está en el inicio mismo. La fecundación artificial desvirtúa el sentido de la maternidad y la paternidad, al ponerlas en un contexto técnico y comercial donde pueden surgir problemas como en todos los contratos, operaciones mercantiles y prestación de servicio con precio" (Serrano, 2006, pág. 1). Añade que si no se quiere replantear todo, al menos podría hacerse el sistema un poco menos absurdo, dando en primer lugar alguna relevancia jurídica al interés del embrión, pues no tiene sentido que la ley -como ocurre en Gran Bretaña- antes de admitir una solicitud de reproducción asistida considere el bien del niño que nacerá y una vez que es embrión ya no lo considere; por otro lado, se reducirían los pleitos si el acceso a la reproducción asistida se limita a los matrimonios (así es en algunos países) y si el consentimiento fuere irrevocable desde el primer momento; ello además de permitir crear solo los embriones estrictamente necesarios para su uso y prohibiendo la posibilidad de congelarlos, a fin de evitar dar tiempo a que cambien circunstancias o voluntades (Ibid., pag. 3). 
drome de Down y una grave afección en el corazón. La noticia generó una gran indignación a nivel internacional; y hasta el propio primer ministro de Australia se hizo partícipe del caso y ofreció ayuda para el niño abandonado -llamado Gammy- cuya concepción había sido "encargada" por 10 mil euros a Pattaramon Chanbua, una mujer de 21 años, de escasos recursos y con muchas deudas. Conocido el problema durante el embarazo, la pareja australiana le había pedido que abortara, a lo que ella se negó porque el aborto no es aceptado por el budismo, su religión.

No es éste, por cierto, el primer caso de este tipo. A raíz del caso de Gammy, se han conocido otros como el de Delaney Ott-Dahl, en Estados Unidos, una niña nacida con síndrome de Down, que también fue rechazada por quienes contrataron a su madre mediante un contrato de maternidad subrogada. ${ }^{12}$

Asimismo, en marzo de 2013, un reportaje de la CNN reveló el caso de una pareja de Connecticut que ofreció a la madre subrogada que había contratado para gestar un bebé, nada menos que la suma de US\$10,000 para que aborte el niño que estaba gestando por considerarla la "solución más humana" debido a que venía con una serie de defectos físicos (quiste en el cerebro, pa- ladar hendido y una anormalidad compleja en el corazón), instándola a que termine con el embarazo de inmediato. La madre -Crystal Kelley-que en un momento llegó a pedir US 15.000 , pero finalmente se negó y continuó con el embarazo, marchándose a Michigan para no tener problemas legales, pues en dicho estado se considera como madre a la madre subrogada; luego, nacida la niña, buscó un programa para dar en adopción a su bebé, lo cual consiguió con éxito.

En octubre de 2010, asimismo, salió a la luz pública el caso de una pareja en Canadá que instó a la madre de alquiler que habían contratado, para que abortara el feto que estaba gestando por tener el síndrome de Down. ${ }^{13}$

En el Perú, en el año 2010, se conoció también otro caso que puso sobre el tapete el tema de la fecundación in vitro: Una pareja chiclayana los esposos A. Rodríguez y W. Gonzáles- anunciaron públicamente que demandarían a la clínica Concebir por un millón de soles, debido a haber tenido una niña con síndrome de Down y con males cardíacos congénitos, luego de que se sometieran a una fecundación in vitro en dicho lugar y tuvieran mellizas. Los padres argumentaron que ellos se sentían engañados al recibir un "producto fallado" y cues- tionaron que no se haya hecho previamente un Diagnóstico Genético Preimplantacional (DGP) para seleccionar otro embrión, acusando a la clínica de negligencia médica. ${ }^{14}$

La pareja expresó públicamente que se sentía estafada por haber pagado US\$15.000 por el tratamiento completo de fecundación in vitro, habiéndoles prometido la clínica "los mejores embriones: los más vivos, los más capacitados" y no les habían cumplido; en un reportaje, incluso, el padre preguntó al entrevistador: "¿Cómo se sentiría si le dieran un producto fallado?"

\section{Algunos cuestionamientos a LA FECUNDACIÓN IN VITRO}

La instrumentalización muchas veces subyacente de la mujer, la comercialización de la técnica, la manipulación y pérdida de embriones que ella implica, la aplicación del diagnóstico pre-implantatorio cual control de calidad, la discriminación de los embriones defectuosos que lleva a "desecharlos", lo artificial de la técnica al evadir la selección natural con los riesgos de taras - enfermedades genéticas que implica, el hecho que su aplicación en realidad no remedia la esterilidad de las parejas, el mal uso de las técnicas por mera comodidad o por capricho, entre otras razones, llevan a la

12 https://www.yahoo.com/health/california-couple-shares-surrogate-story-in-wake-of-95207128652.html (Consultada el 30.08.2014).

13 http:/ / life.nationalpost.com/2010/10/06/couple-urged-surrogate-mother-to-abort-fetus-because-of-defect / (Consultada el 31.08.214C)

14 Diario El Comercio. Lima: edición del 12.11.2010. En: http://elcomercio.pe/lima/667840/noticia-padre-bebe-in-vitrocomo-se-sentiria-si-le-dieran-producto-fallado (Consultada el 30.12.2010). 
crítica de las mismas, como enumera Morán de Vicenzi. ${ }^{15}$

También el profesor nacional Víctor Guevara Pezo, luego de pronunciarse porque solo se debe de aceptar la inseminación homóloga, manifiesta que el uso de la fecundación in vitro es objetable por la supresión de varias vidas que implica la selección de embriones y por cuanto considera que "... consagra la deshumanización de la relación humana de los seres, pues ésta es reemplazada por la intermediación manipuladora de los laboratorios que pretenden convertirse en factorías de vida" (Guevara Pezo, 2002, pág. 140). ${ }^{16}$

Otro factor que se menciona -frecuentemente obviado- es que, en el caso de la subrogación materna, "además surgen, graves problemas en consideración del "diálogo" materno-fetal que se instaura desde las primeras fases del desarrollo del embrión, demostrado indubitablemente desde el punto de vista científico". ${ }^{17}$

Sobre las consecuencias de recurrir a las técnicas de reproducción asistida, manifiesta por su parte Antonio Carrera:

“... implica procesos largos y penosos. Es un camino de decepciones y esperanzas que puede implicar varios años de la vida de la mujer y de su pareja (...) La presión psicológica a la que se enfrenta la pareja, en especial la mujer, es muy desgastante, pues el procedimiento (...) está lleno de inquietudes, sufrimientos, angustias, miedos, además de soportar los efectos de los medicamentos y enfrentarse a procedimientos fuertemente invasivos. El hombre, por su lado, se siente desplazado, debido a que el protagonista principal es el médico que atiende a su esposa y será el que haga posible que su esposa quede embarazada" (Cabrera, 2011, pág. 34).

En cuanto a las consecuencias de tipo psicológico, estima Cabrera que la mujer puede subvalorar a su esposo en razón del donador o al revés. Puede aparecer un sentimiento de haber traicionado al esposo y otros sentimientos de culpa y agresividad en sus relaciones, además de la posibilidad de generar en el marido sentimientos de inferioridad. (Cabrera, 2011, pág. 34).

Vale decir que, en el caso de la reproducción asistida homóloga, la tendencia ha sido la de una cada vez más creciente aceptación en los ordenamientos jurídicos; apareciendo como más polémica; sin embargo, la reproducción asistida heteróloga. Entre los argumentos en contra, se menciona, que en tal supuesto intervienen componentes genéticos extraños (al marido, a la mujer o a ambos), asumiéndose el criterio de que ningún ser humano tiene derecho, desde un punto de vista ético, a disponer de sus componentes genéticos (Cárdenas Quirós, 1994, pág. 85), por lo menos de esta manera. Vila-Coro, por su parte, afirma que

"La reproducción asistida heteróloga modifica la trayectoria vital del individuo, le priva de su derecho de ser la consecuencia de su propia indeterminación. Además, incide en su independencia, entendida la independencia como expresión de libertad, al insertarle en un medio familiar que le es ajeno (Vila-Coro, Los límites de la Bioética, pág. 71).

Ante el argumento que invoca la autonomía de la voluntad para justificar el uso de esta técnica, sostiene Ana María Vega:

“Es fácil y cómodo adu-
cir el libre desarrollo de
la personalidad como ar-
gumento para justificar
el máximo respeto a las
decisiones procreativas.
Pero la libertad exige
responsabilidad, espe-
cialmente cuando en el
ejercicio de esa liber-
tad están implicados los
derechos fundamentales
de otras personas, en
este caso, los del futuro
hijo"18

15 Para Claudia Morán estas técnicas no deben aplicarse por ser ilícitas al vulnerar el derecho a la vida y a la dignidad de los concebidos (Morán de Vicenzi, 2008, pág. 154).

16 Guevara Pezo, Víctor (2002). "Vacíos en el sistema legal en materia de biojurídica". En: Varios autores. Bioética y Biojurídica. La Unidad de la Vida. Lima: Ediciones Jurídicas UNIFE, p. 140.

17 Torlone, Gaetano (2010). “La familia y Bioética”. En: Apuntes de Bioética, Año 1, No. 1, setiembre 2010, p. 63.

18 Vega, Ana María. "El derecho a un hijo". En: http://www.aceprensa.com/articles/el-derecho-a-un-hijo/ (Consultada el 12.12.2013). 
En todo caso, frente a la práctica de la misma, es fundamental que la aplicación de la técnica vaya aparejada de una adecuada información a quienes recurren a ella, a fin de garantizar un efectivo consentimiento informado, como también la transparencia del caso. Por ejemplo, tenemos entre nosotros que existen institutos y clínicas que facilitan la reproducción asistida, pero no se tiene ninguna referencia de dónde obtienen los espermatozoides 0 los óvulos que utilizan, sin contar con que ofrecen incluso "servicios" de "ovodonación" o de selección de sexo, pese a que jurídicamente ello no está permitido. ${ }^{19}$

Por otro lado, es de tener presente que el éxito de estas técnicas es estadísticamente bajo, además de ser un tratamiento oneroso y largo, de impredecibles resultados, lo cual debería ser informado debidamente a las personas interesadas, para no crearles falsas expectativas.

Sobre la posibilidad que una mujer sola pueda hacer uso de los métodos de reproducción asistida, juristas como el argentino Gustavo Bossert se oponen invocando el principio del interés superior del niño, ya que se estaría trayendo al mundo exprofesamente un niño sin padre; también se opone a la idea Rodolfo Vásquez, teniendo en cuenta la necesidad del niño de contar con una familia estable, entre otras razones (Vásquez, 1993). Claro que es cierto que lo mismo pasa cuando una mujer enviuda durante su embarazo, pero la diferencia es que mientras en un caso se trata de un hecho impredecible, en el otro se trata de algo que podemos evitar.

Bossert hace un interesante análisis del tema, a partir de su comparación con la adopción:

Así como en la adopción una mujer sola (sea soltera, divorciada o viuda), puede incorporar a su vida como hijo, a un niño extraño, parece que igual podría hacerlo por la vía biológica (y -agregamos- podría pensarse entonces en la aplicación del principio "donde hay la misma razón, hay el mismo derecho"). Pero debe advertirse entonces una diferencia esencial si vemos el caso desde la perspectiva del niño, pues, en el caso de la adopción, la mujer incorpora a su vida una criatura que ya existe y que seguramente está abandonada, mientras que en el otro caso se está hablando de crear un niño que todavía no existe. Ante esto, la situación óptima para una criatura es acceder a un hogar en donde exista un padre y una madre. "Despojados totalmente de prejuicios, sólo por esta perspectiva del interés de la criatura, es que nos parece que el texto legal debería excluir la posibilidad de la inseminación de la mujer sola" (Bossert, 1995, pág. 91).

También son temas discutibles que giran en torno a las técnicas de reproducción asistida: el contrato de "alquiler" de vientre, la discusión de la posibilidad de aplicar estas técnicas en el caso de concubinos, el caso de error en el uso del material genético (Bossert, 1995, pág. 89), etc., pero, en todo caso, estimamos que, en general, en cuanto a las técnicas de reproducción asistida, debemos tener presente las palabras del citado autor, quien luego de considerar a las mismas, junto a la fisión del átomo y la salida al espacio, como los logros científicos del siglo XX que, de seguro, van a incidir más hondamente en el futuro de la humanidad, agrega que: "Cada uno de ellos ofrece posibilidades notables de contribuir al bienestar, pero también

19 En Inglaterra, en noviembre del año 2003, la Autoridad de Embriología y Fertilización Humana (HFEA, por sus siglas en inglés), rechazó la libre elección del sexo de los bebés, salvo razones estrictamente médicas (ej. Caso de hemofilia); la iniciativa fue aprobada teniendo en cuenta la opinión del $80 \%$ de la población en contra de la técnica, estimando que se debe valorar la naturaleza incondicional del amor de los padres. Empero, países como Chipre, Estados Unidos, Jordania, México, Nigeria, Panamá, República Checa y Tailandia, sí permiten actualmente la selección del sexo. La Ley sobre Técnicas de Reproducción Asistida española considera entre sus infracciones "muy graves" la selección de sexo, con la única excepción de la existencia de motivos terapéuticos.

20 La propuesta recomienda que la palabra "parent" sea reemplazada por "other significant adults" u "other people of significance to the child", omitiéndose hablar entonces de "both parents", pues ya no tendría sentido hablar de "ambos padres", al reconocerse distintas categorías de paternidad: legal, adoptiva, genética, intencional, psicológica, social y subrogada, entre otras (Boletín BioEdge. Australia: edición del 17 de agosto de 2014. En: http://www.bioedge.org/index. php/bioethics/bioethics_article/11107. (Consultada el 17.08.2014). 
a la desviación y la catástrofe, si no prevalece la prudencia en la aplicación y perfeccionamiento de esas conquistas" (Bossert, 1995, pág. 89).

Para Espinoza, en cambio, debería permitirse someterse a este tipo de prácticas a las parejas, casadas o no y a las mujeres solteras en edad fértil (Espinoza Espinoza, 2012, pág. 129).

El desarrollo de la reproducción asistida ha generado, entre otras cosas, que, en agosto del 2014, se haya planteado en Australia la posibilidad de revisar el ordenamiento jurídico para permitir el reconocimiento de una paternidad múltiple, según un informe preparado para el gobierno, denominado "Report On Parentage And The Family Law Act"; la propuesta se basa en que la adopción y las nuevas formas de reproducción a través de la tecnología están creando nuevas tensiones en la definición del concepto de paternidad. ${ }^{20}$

\section{Consentimiento ¿informa- DO?}

Siendo obligatorio el consentimiento informado, muchas de las personas que se someten a técnicas de fecundación asistida, no suelen estar debidamente informadas tanto de los riesgos que pueda sufrir el menor, como de los riesgos que pueda sufrir la misma madre, por lo que es importante abordar el tema a partir de la lectura especializada en revistas científicas norteamericanas y europeas que constituyen fuentes primarias de primer nivel, de las que no se suele dar cuenta en el tratamiento del tema en nuestros predios jurídicos.

\section{Riesgos para lOS NIÑOS NA- CIDOS MEDIANTE TÉCNICAS DE REPRODUCCIÓN ASISTIDA}

Cuando se habla de la fecundación asistida en nuestro medio, la cuestión suele reducirse a resaltar los beneficios de la técnica, a una somera revisión legal, o a la mención de lo anecdótico, obviándose cuestiones médicas y científicas fundamentales, que pasamos a exponer.

Un estudio científico realizado en Dinamarca, evaluando 2.166 casos, publicado en la revista Human Reproduction, arrojó como resultado que el riesgo de que un niño concebido mediante reproducción asistida nazca muerto es del 16,2 por mil, siendo este porcentaje en el caso de reproducción natural del 2,3 por mil (Wisburg, K., Ingerslev, $\mathrm{HJ}$, Henriksen, TB, 2010). ${ }^{21}$

Por otro lado, en un estudio del que da cuenta la revista Fertility and Sterility, sobre los resultados de las técnicas de reproducción asistida en Canadá, en el año 2006, se evaluaron 12.052 ciclos de fecundación in vitro, observándose que el índice de embarazos por ciclo iniciado fue de $33,7 \%$ y el de niños nacidos vivos de 27,1\% (Gunby, Joanne; Bissonnettte, Francxois; Librach, Clifford y Lisa Cowan., 2010). Al año siguiente, el índice de embarazos por ciclo fue de $35.6 \%$ y el de niños nacidos vivos fue de $28.6 \%$ (Gunby, Joanne; Bissonnette, Francxois; Librach, Clifford and Lisa Cowan, 2011).

Otro estudio, publicado por la revista científica Obstetric and Gynecology, de The American College of Obstetricians and Gynecologists, reveló que todos los embarazos en los que se ha recurrido a tecnologías de reproducción asistida están asociados no solo con embarazos múltiples y necesidad de recurrir a cesáreas, sino también con anormalidades congénitas (Buckett, William; Chian, Ri-Cheng; Holzer, Hananel; Dean, Nicola; Usher, Robert y Seang Lin Tan, 2007).

En adición a lo expuesto, cabe mencionar que la revista científica Human Reproduction, publicó el año 2008, un estudio mediante el que se comprueba que los niños nacidos de embriones congelados, obtenidos tanto por fecundación in vitro como por inyección citroplasmática de espermatozoides (ICSI), tienen mayor índice de malformaciones que los nacidos de embriones no congelados (Belva, F.; Henriet, S.; Van den Abbeel, E.; Camus, M.; Devroey, P.; Van der Elst,

\footnotetext{
1 Dentro de las conclusiones del estudio, se señala lo siguiente: “Compared with fertile women, women who conceived by IVF/ICSI had an increased risk of stillbirth that was not explained by confounding. Our results indicate that the increased risk of stillbirth seen after fertility treatment is a result of the fertility treatment or unknown factors pertaining to couples who undergo IVF/ICSI" (Para más detalle, véase: http://www.medscape.com/viewarticle/721241 o http://www.ncbi. nlm.nih.gov/pubmed/20179321).
} 
J.; Liebaers, I.; P. Haentjens and $M$. Bonduelle, 2008). ${ }^{22}$

Por otro lado, en Inglaterra, conforme da cuenta The New England Journal of Medicine, otro estudio confirma el mayor riesgo de nacimiento con defectos de los concebidos mediante reproducción asistida en comparación con los nacidos mediante la concepción espontánea (Davies, Michael J.; Moore, Vivienne M.; Willson, Kristyn J.; Van Essen, Phillipa; Priest, Kevin; Scott, Heather; Haan, Eric A. Haan and Annabelle Chan, 2012). ${ }^{23}$

Además, conforme da cuenta Xavier Simmons, un estudio hecho por investigadores australianos sobre la base de niños nacidos mediante reproducción asistida entre 1995 y 2012, reveló que estos son más proclives a tener defectos de nacimiento en un $32 \%$. Detrás de ello, se mencionan factores asociados con el tratamiento que podrían incrementar el riesgo en nacimientos, incluyendo las mismas causas de infertilidad subyacentes, las propias técnicas de reproducción asistida, como los medicamentos usados; cuestiones vinculadas con el congelamiento y descongelamiento de los embriones, alteraciones hormonales al tiempo de la implantación, la manipulación de gametos y embriones o una combinación de estos (Symons, 2013, pág. 7).

También es de mencionar que, en abril del 2012, se conoció la noticia que dos recientes estudios han mostrado que la incidencia de defectos de nacimiento y problemas circulatorios a largo plazo son más probables en niños concebidos mediante una fecundación in vitro. Lo primero ha sido señalado por investigadores del Nanjing Medical University de China, cuyo trabajo ha sido publicado en la revista Fertility and Sterility (Wen, Juan; Jiang, Jie; Ding, Chenuye; Dai, Juncheng; Liu, Yao. Xia, Yankai; Liu, Jiayin y Zhibin Hu., 2012). A conclusión similar ha llegado en EEUU el Center for Disease Control and Prevention.
Las razones de por qué hay mayores problemas de salud en los niños nacidos mediante técnicas de reproducción asistida, se ha buscado explicarlas acudiendo a diversas teorías:

a) Las personas que tienen problemas para concebir tienden a tener bebés con defectos de nacimiento, justamente debido a esos mismos problemas, vinculados con los gametos de los progenitores, y no a las técnicas de reproducción asistida en sí.

b) Razones relacionadas con la aplicación del tratamiento.

c) Cuestiones vinculadas tanto con el estado de los gametos como las técnicas empleadas causan los trastornos en la descendencia con distinta influencia según el tipo de entorno (López Moratalla, Natalia; Huerta Zepeda Alejandra y Dolores Bueno López, 2012/2, pág. 469). ${ }^{24}$

22 El problema, aparte de las malformaciones en sí, es que no se informa a las personas que recurren a estas técnicas, el hecho de la existencia de ese mayor riesgo; un estudio hecho en México, por ejemplo, concluye lo siguiente: "se puede concluir que los porcentajes de desinformación para los grupos que se sometieron a alguna de las TRA son elevados y, de manera especial, en cuanto a las probabilidades de malformaciones genéticas en los embriones"; en una escala entre nada, poco, mucho o muchísimo, dijeron no saber nada 58.3\% de los que se sometieron solo a una inseminación artificial, 42.7\%. de los que se sometieron a inseminación artificial y FIVET-ICSI y $57.1 \%$ de los que se sometieron a solo FIVET y/o ICSI (Cabrera, 2011, págs. 152-153).

23 Señalan los referidos autores: "We confirmed previous findings of an increased risk of birth defects among births conceived with assisted reproductive technology as compared with births from spontaneous conception". Agregan luego que: "Women in the assisted-conception group were also more likely to have a stillbirth and to deliver by cesarean section and at a gestation of less than 37 weeks or less than 32 weeks and were less likely to have a male singleton (Table 2). In addition, their children had a lower mean birth weight than the children of women in the spontaneous- conception group" (pag. 1805). Births after any assisted conception were associated with a significantly increased risk of any birth defect: $8.3 \%$, as compared with births to fertile women that did not involve assisted conception: $5.8 \%$ (pag. 1808).

24 "El desarrollo de las condiciones de cultivo de los embriones en el laboratorio, y en su caso la congelación, por mucho que se haya perfeccionado con la experiencia a posteriori, no sustituye al entorno natural preciso, y el único que evita la vulnerabilidad del embrión precoz" (Ibid.: 471). "La sustitución, imprescindible cuando se usa las TRA, del medio natural por el medio in vitro puede causar mutaciones en este nivel de regulación -epimutaciones- que se suman a las posibles alteraciones, que de suyo o por la manipulación técnica, puedan tener los gametos de los progenitores. Una concatenación de causas conlleva consigo el hecho de que la salud de los que llegan a nacer mediante el uso de las TRA será peor que la de los nacidos que fueron engendrados. Más aún, algunas de las epimutaciones del ADN pueden pasar a las siguientes generaciones" (Ibid: pag. 479). 
d) En realidad, no es que hayan más defectos, sino que ellos son más conocidos hoy en día simplemente porque estos niños están más cercanamente observados.

Desde que nació Louise Brown en 1978, el Medical Research Council empezó a comparar los datos del primer año de vida de los niños nacidos mediante fecundación in vitro durante diez años, respecto a los niños nacidos en el mismo período naturalmente, encontrándose ya desde entonces un déficit de salud de los generados por la fecundación in vitro; luego, hacia los años 2003-2005, se realizan meta-análisis que recogen datos de diversos estudios, que exponen un incremento del riesgo por la aplicación creciente de las técnicas (Ibid., pag. 469).

Señalan López Moratalla, Huerta y Bueno que ya a inicios de 1990 se había dado una alerta pediátrica debido a los defectos y anomalías con que nacían varios de estos niños concebidos por fecundación asistida, cuya peor salud se evidencia a partir del 2002; los problemas tienen que ver con que la técnica implica la pérdida de varios embriones antes de su implantación, abortos espontáneos, alta mortalidad perinatal, no se ha hecho previamente la imprescindible experimentación con animales, las pruebas hechas con animales fértiles han mostrado que las crías generadas in vitro presentan alteraciones graves; se ha hecho una extensa experimentación humana directa sin los requisitos mínimos de la experimentación humana, sin saber el papel de las técnicas en sí y cómo causan los efectos que ellas generan, etc. (Ibid., pag. 477-478).

Aparte de este riesgo, la aplicación de las técnicas de reproducción asistida "generan síndromes raros, y aparecen casos recientes que muestran alteraciones no cuantificadas aún", agregando López Moratalla, Huerta y Bueno (2012) que: "Conocemos que hay alteraciones que aparecen a largo plazo, como la enfermedad sistémica pulmonar y cardiovascular, causadas por la exposición del embrión en los primeros días -en los que es especialmente vulnerable-, a un entorno adverso y a la estimulación ovárica”.

Observan los mismos autores que "a lo largo de la década de los 90 se observa una clara relación en la aplicación de la FIV, y la prematuridad y bajo peso de los niños al nacer. Se describe hipertensión, enfermedades cardíacas y osteoporosis y un mayor grado de malformaciones" (Ibid.: pag. 485).
Los resultados del estudio de M. Hansen y otros, publicado en el 2005, en The New England Journal of Medicine sugieren que existe un aumento del riesgo estadísticamente significativo de defectos de nacimiento en los bebés concebidos mediante técnicas de reproducción asistida del orden de 30-40\%; mayores riesgos que deberían ser informados a toda persona que desee someterse a este tipo de tratamientos. ${ }^{25}$

Todo ello revela que, luego de más de 35 años de investigación, todavía no tenemos el control necesario ni el conocimiento suficiente de las técnicas de reproducción asistida.

Se señala como motivos muy claros que, como no se cura la esterilidad, el hijo podrá padecer las consecuencias de las deficiencias de los gametos de los padres. Pero, incluso si el problema no es que los gametos sean defectuosos, como se da cuando se recurre a donantes, la aplicación de las técnicas tiene excesivas deficiencias, como señalan López Moratalla, Huerta y Bueno (2012/2, pag. 474). Agregan también, citando a diversos autores, que, respecto a la parálisis cerebral, "Diversos estudios desde 2006 han afirmado que los niños nacidos tras FIV o ICSI tie-

25 Klerk, N. y Kurinczuk, J.J., 2005). Concluye el estudio señalando expresamente que "Pooled results from all suitable published studies suggest that children born following ART are at increased risk of birth defects compared with spontaneous conceptions. This information should be made available to couples seeking ART treatment". Cabe señalar que, cinco años antes, el estudio de algunos de los mismos investigadores había dado el alerta de que los niños concebidos mediante la tecnología de reproducción asistida tenían más de dos veces que los niños concebidos naturalmente, defectos de nacimiento detectados durante el primer año de vida y tenían mayor predisposición a tener otros problemas (Hansen, M.; Kurinczuk, J.J.; Bower, C. y Webb, S., 2002, pág. 729). Es de agregar la siguiente consideración del mismo estudio publicado en el New England Journal of Medicine: "We found that there may be an excess occurrence of major cardiovascular, urogenital, chromosomal, and musculoskeletal defects associated with assisted conception. However, these findings regarding specific organ systems should be interpreted with caution, since they are based on small numbers of infants in each group" (Ibid. p. 730). 
nen mayor riesgo de sufrirla; además presentan mayor prevalencia en el retraso mental y la disfunción ocular severa asociados a esta enfermedad, así como trastornos del espectro del autismo" (Ibid., 489-490). Añaden a ello la vascularización anormal de la retina y anomalías cardíacas.

En una carta dirigida a la Canadian Fertility and Andrologic Society, la genetista de la Universidad de Toronto, Dra. Rosanna Weksberg, sostiene que los bebés nacidos mediante una fertilización in vitro, tienen 10 veces más posibilidades de sufrir algunos raros desórdenes de carácter genético, por lo que deben hacerse más estudios sobre materia. Estos problemas, aparte del bajo peso, incluyen un mayor riesgo de autismo. ${ }^{26}$

Por otro lado, los resultados de un estudio realizado por el grupo del Hospital Clínic-Idibaps (Instituto de Investigaciones Biomédicas August Pi i Sunyer) de Barcelona, publicado en la revista Circulation en el año 2010 y del que diera cuenta el boletín Provida Press, revela que aproximadamente la mitad de los niños concebidos con técnicas de reproducción asistida tienen riesgo de padecer en un futuro de enferme- dades cardiovasculares, frente a un $5 \%$ en el caso de los bebés que han nacido producto de una concepción natural.

En este listado de riesgos, anota María Lacalle que "estudios recientes parecen demostrar que las técnicas de reproducción asistida producen una serie de efectos directos sobre el genoma de los embriones. Se trata de modificaciones epigenéticas por metilación de bases nucleotídicas. La incidencia tumoral en los niños así concebidos es muy superior a la media" (Lacalle Noriega, 2013, pág. 120).

Se dice también que quienes nacen de una fecundación in vitro, tienen el doble de posibilidades de morir en los primeros 28 días luego del nacimiento que los nacidos por vía natural, además de sufrir de prematuridad y aumentar hasta tres veces la posibilidad de nacer con bajo peso, según da cuenta la publicación Plos One en su edición de enero del 2014. ${ }^{27}$

\section{RIESGOS CON RELACIÓN A LA SALUD DE LA MADRE}

No puede tampoco dejarse de mencionar que la fecundación in vitro no solo podría tener algunos efectos en el niño, sino que también pueden existir algunos riesgos en cuanto a la salud de la madre; así, conforme mencionan López y Abellán, "hasta un 10\% de las mujeres que se someten a estas técnicas desarrollan el síndrome de hiperestimulación ovárica severo que puede causar dolor, fallo renal, potencial infertilidad futura, ascitis, tromboembolia, e incluso la muerte" (López Barahona, Mónica y Abellán, José Carlos, 2009, pág. 25) . ${ }^{28}$

El estudio de campo hecho por Antonio Cabrera, nos da una perspectiva integral de la problemática, la cual involucra aspectos psicosomáticos:
"el principal riesgo físico para la mujer, si se somete a altas dosis de hormonas 0 si se somete a tratamien- tos repetitivos del mismo tipo, es la posibilidad de serios trastornos de coagu- lación como tromboembolia o el síndrome de hiperesti- mulación ovárica, (...). De- bido a la repetición y agre- sividad de los procedimien- tos, a la ansiedad con que se esperan los resultados, a los tratamientos hormona- les, la mujer puede sufrir física y psicológicamente y verse reducida a la condi- ción de un simple mecanis-

26 http://www.lifesitenews.com/news/ivf-babies-up-to-10-times-more-likely-to-suffer-rare-genetic-disorders-gene. (Consultada el 22.08.2014).

27 Plos One; 2014; 9, No.1, e80398, enero 2014, citado por el Observatorio de Bioética de la Universidad Católica de Valencia San Vicente Mártir. En: http://www.observatoriobioetica.org/2014/riesgos-medicos-de-la-fecundacion-invitro/?utm_source=wysija\&utm_medium=email\&utm_campaign=429.

28 Agregan López y Abellán que, según datos provenientes de investigaciones con animales (aún no se tienen estudios concluyentes en humanos) existen sospechas fundadas de que las mujeres que se someten a múltiples ciclos de hiperestimulación ovárica puedan tener mayor riesgo de padecer cáncer de ovario o mama (Ibid., p. 26). Dentro de los riesgos de recurrir a la técnica de hiperestimulación hormonal a la que debe someterse una mujer que desea donar sus óvulos, menciona Justo Aznar que ellos "pueden variar desde el dolor abdominal, náuseas, vómitos, e incluso otros más graves como aumento de peso, dificultades respiratorias y efectos dañinos en otros órganos como vejiga, intestino o útero, a la vez como daños indirectos a más largo plazo como disminución de la fertilidad, incluso infertilidad, hemorragias, riesgo tromboembólico, incluso aumentar el cáncer de ovario, pecho y colon, e incluso en muy raras ocasiones la muerte". 
mo reproductor" (Cabrera, 2011, pág. 33).

\section{Consideraciones finales}

Frente al cada vez mayor uso, abuso y riesgos de las técnicas de reproducción asistida ${ }^{29}$, resulta oportuno incidir en revisar la figura de la adopción como una figura alternativa a tener presente, teniendo en cuenta que, como bien señala Ana María Vega: "La adopción, a diferencia de la procreación asistida, no crea huérfanos, sino que remedia las condiciones de vida de lo que ya están en el mundo". ${ }^{30}$

Al amparo de la libertad, que nos ha permitido desarrollarnos, es muy importante apelar a la tecnología para mejorar nuestra existencia, pero frente al frenesí con que nos solemos entregar a las novedades y a las tentaciones de la tecnología, es importante asumir una actitud fronética que nos haga detenernos a pensar en qué es lo que estamos haciendo, qué es lo que queremos hacer, y determinar si ese camino es el correcto.

"Es necesario preguntarse si es posible detener o incluso dar marcha atrás al avance que han tenido las TRA en los últimos años. El escenario actual, a nivel mundial, parece indicar que esto no será posible y que ocurrirá todo lo contrario: el número de clínicas de infertilidad irá en aumento y los tratamientos realizados en dichas clínicas, también. Por ello, es ineludible efectuar una reconsideración de las TRA a fin de redescubrir, entre otros temas, el sentido de la generación humana (...), del curar (...) $e$ insistir más en prácticas que conlleven un auténtico diagnóstico de las causas de la infertilidad" (Cabrera, 2011, pág. 38).

Vistas objetivamente las cosas, parece imparable el desarrollo de las técnicas de reproducción asistida, por lo que, a la vez de seguir investigando para poner en evidencia sus riesgos, es necesario encausar dicho desarrollo en el marco del respeto a la dignidad, la integridad y la identidad del ser humano.

Ahora bien, nos encontramos en un momento en el que -como dice Corral-, "el Derecho debe responder a un momento histórico de intenso cambio en la familia y en la esfera de la reproducción" (Corral García, 2013/2a, pág. 39) , lo cual demanda una actitud atenta y militante en la defensa de valores y principios fundamentales.

Las técnicas de reproducción asistida surgieron para ayudar a matrimonios estériles para lograr tener un bebé, mas hoy en día su uso parece no solo haberse distorsionado sino que también se ocultan algunos riesgos en torno a ella, tanto para el concebido como para la madre.

En esta materia, debe recordarse que no hay libertad sin responsabilidad. Tampoco puede hablarse sin error de que exista un "derecho al hijo", pues éste no es un objeto apropiable, ningún ser humano puede ser cosificado, y no existe declaración internacional de derechos que reconozca este pretendido "derecho".

La trascendencia de la libertad está en que ella se ejerce en función de valores, éticamente, entendiendo a la ética como un pensar en el otro, afirmando nuestra "mismidad" como seres humanos en ese pensar en el otro.

Sin que se trate de un derecho absoluto legalmente, las personas son libres de recurrir al empleo de las técnicas de reproducción asistida, dentro de los términos que determina la Ley General de Salud en su artículo 7, teniendo en cuenta el mandato constitucional de proteger a la familia, así como el principio del interés superior del niño y demás normas pertinentes; debiendo cumplirse siempre con que cualquier decisión que se tome respecto a la persona relacionada con su salud e integridad, cuente con su consentimiento informado, lo cual implica, en este caso, decirle claramente al paciente, junto con los beneficios, los riesgos propios de la fecundación asistida, tanto para el hijo como

\footnotetext{
29 "La prohibición de la fecundación heteróloga es exactamente debida al respeto de los "derechos ajenos", o bien del derecho del concebido a tener un solo padre y una sola madre al mismo tiempo bajo los perfiles genético, afectivo y legal" (Casini, 2012/4, pág. 478) .

30 Vega, Ana María. "El derecho a un hijo”. En: http://www.aceprensa.com/articles/el-derecho-a-un-hijo/ (Consultada el 12.12.2013).
} 
para la madre, a fin de poder tomar las decisiones más correctas, con la mayor prudencia, justicia y sabiduría.

\section{REFERENCIAS BIBLIOGRÁFICAS}

Belva, F.; Henriet, S.; Van den Abbeel, E.; Camus, M.; Devroey, P.; Van der Elst, J.; Liebaers, I.; P. Haentjens and M. Bonduelle (2008). "Neonatal outcome of 937 children born after transfer of cryopreserved embryos obtained by ICSI and IVF and comparison with outcome data of fresh ICSI and IVF cycles". En: Human Reproduction; 23 (10); p. 2227-2238.

Bossert. Gustavo (1995). "Fecundación humana asistida". En: Varios autores. Derecho Civil de nuestro tiempo. Lima: Universidad de Lima - Gaceta Jurídica.

Buckett, William; Chian, RiCheng; Holzer, Hananel; Dean, Nicola; Usher, Robert y Seang Lin Tan (2007). "Obstetric Outcomes and Congenital Abnormalities After In Vitro Maturation, In Vitro Fertilization, and Intracytoplasmic Sperm Injection". En: Obstetrics \& Gynecology; October 2007 - Volume 110 - Issue 4 - pp 885-891.

Cabrera, Antonio (2011). La atención integral a las personas con problemas de fertilidad: Estudio de campo realizado en México. Tesis de Doctorado para el Ateneo Pontificio Regina Apostolorum, presentada en Roma. México.
Cárdenas Quirós, Carlos (1994). Estudios de Derecho Privado (Reflexiones de un tiempo). Lima: Ediciones Jurídicas.

Casini, M. (2012) "Tres Tribunales Italianos, la Corte Europa de los Derechos de los Hombres y el derecho del concebido a la individualidad de las figuras paternas". En: Medicina y Ética. Revista Internacional de Bioética, Deontología y Ética Médica. Vol. XXIII, Número IV 2012/4, Oct.-Dic. 2012. México: Instituto de Humanismo en Ciencias de la Salud - Facultad de Bioética - Univ. Anáhuac e Istituto di Bioetica - Facoltá de Medicina e Chirurgia "Agostino Gemelli” - Universitá Cattolica del Sacro Cuore - Roma.

Corral García, Eduardo (2013). “El lenguaje bioético en la normativa y jurisprudencia sobre problemas biojurídicos". En: Cuadernos de Bioética XXIV 2013/2a. Madrid: Asociación Española de Bioética y Ética Médica, AEBI.

De Freitas Drumond, José Geraldo (2012). "Ética, bioética y los desafíos del siglo XXI". En: Derecho No. 69, Revista de la Facultad de Derecho de la Pontificia Universidad Católica del Perú. Lima: PUCP.

Espinoza Espinoza, Juan (2012). Derecho de las Personas. Concebido y Personas Naturales. Tomo I. $6^{\mathrm{a}}$. Edición Lima: Grijley.

Guevara Pezo, Víctor (2002). "Vacíos en el sistema legal en materia de biojurídica". En : Varios autores. Bioética y Biojurídica. La Unidad de la Vida. Lima: Ediciones Jurídicas UNIFE.

Gunby, Joanne; Bissonnettte, Francxois; Librach, Clifford and Lisa Cowan (2010). "Assisted reproductive technologies (ART) in Canadá: 2006 results from the Canadian ART Register". En: Fertility and Sterility; Vol. 93; p. 1312-1316.

Gunby, Joanne; Bissonnette, Francxois; Librach, Clifford and Lisa Cowan (2011). "Assisted reproductive technologies (ART) in Canada: 2007 results from the Canadian ART Register". En: Fertility and Sterility. Vol. 95, No. 2; February 2011; p. 542-547.

Hansen, M. Bower, C.; Millne, E.; de Klerk, N. y Kurinczuk, J.J. (2005) "Assisted reproductive technologies and the risk of birth defects - a systematic review". En: Human Reproduction. 20, 328-338.

Hansen, M.; Kurinczuk, J.J.; Bower, C. y Webb, S. (2002) "The risk of major birth defects after intracytoplasmic sperm injection and in vitro fertilization". En: New England Journal of Medicine, 346, 725-730.

Lacalle Noriega, María (2013). La persona como sujeto de derecho. Madrid: Dykinson.

López Barahona, Mónica y José Carlos Abellán (2009). Los Códigos de la Vida. Madrid: Homolegens. 
López Guzmán, José y Angela Aparisi (2012). "Aproximación a la problemática ética y jurídica de la maternidad subrogada". En: Cuadernos de Bioética, $\mathrm{N}^{\circ}$ 78, vol XXIII, mayo-agosto 2012. Madrid: AEBI

López Moratalla, Natalia; Huerta Zepeda Alejandra y Dolores Bueno López. "Riesgos para la salud de los nacidos por las técnicas de fecundación asistida. La punta de un iceberg". En: Cuadernos de Bioética XXIII, 2012/2. Madrid: AEBI

Morán de Vicenzi, Claudia (2008). "La Filiación y la Fecundación Artificial". En: Varios autores. Temas de Bioética y Derecho. Lima: Cátedra Unesco de Bioética y Biojurídica-Facultad de Derecho de la Universidad Femenina del Sagrado Corazón.

Pérez Monge, Marina (1995). Cuadernos de Bioética 1995/1 ${ }^{\circ}$. Madrid: AEBI.

Rincón Castellanos. Ximena (2012). “¿Bioética y derecho para qué y para quiénes? Discusiones en torno a la regulación de las técnicas de reproducción humana asistida en Colombia". En: Derecho No. 69, Revista de la Facultad de Derecho de la Pontificia Universidad Católica del Perú. Lima: PUCP.

Sambrizzi, Eduardo (2012). "Apuntes varios sobre dis- tintos aspectos de la procreación asistida en el Proyecto de reformas". En: Revista de Derecho de Familia $y$ de las Personas. No, 11. Buenos Aires: La Ley.

Serrano, Rafael (2006). Conflictos que genera la reproducción asistida. En: Aceprensa 031/06 (15.03.2006), tomado de: http.//www.aceb. lorg/tvh06/Evans htm (Consultada el 01.06.2013).

Sgreccia, Elio (2008). A favor de la vida. Temas actuales de Bioética. Buenos Aires: Ágape Libros.

Sunderam, Sasawati; Kissin, Dmitry M.; Crawford, Sara; Anderson, John, E; Folger, Suzanne G.; Jamieson, Denise J. y Wanda D. Barfield (2013). "Assisted Reproductive Technology Surveillance - United States, 2010". En: Morbidity and Mortality Weekly Report (MMWR) / December 6, 2013 / Vol. 62 / No. 9. Atlanta: Centers for Disease Control and Prevention (CDC).

Symons, Xavier (2013). "Birth defects more likely in IVF children". En: BioEdge. Australia, edición del 09.03.2013 (Boletín electrónico).

Torlone, Gaetano (2010). "La familia y Bioética". En: Apuntes de Bioética, Año 1, No. 1, setiembre 2010.

Vásquez, Rodolfo (1993). “Ética, Derecho y Fecundación Asistida". En: Revista Doxa:
Cuadernos de Filosofía del Derecho, No.19. Alicante: Universidad de Alicante.

Vega, Ana María. "El derecho a un hijo". En: http://www. aceprensa.com/articles/elderecho-a-un-hijo/ (Consultada el 12.12.2013)

Vila- Coro, María Dolores (1997). Huérfanos biológicos. El hombre y la mujer ante la reproducción artificial. Madrid: Ediciones San Pablo.

Vila-Coro, María Dolores (1995). Introducción a la Biojurídica. Madrid: Universidad Complutense.

Vila-Coro, María Dolores (1992). "Los límites de la Bioética”. En: Varios autores. Biotecnología y futuro del hombre: la respuesta bioética. Madrid: Eudema, S.A. (Ediciones de la Universidad Complutense S.A.).

Wen, Juan; Jiang, Jie; Ding, Chenuye; Dai, Juncheng; Liu, Yao. Xia, Yankai; Liu, Jiayin y Zhibin Hu. (2012) "Birth defects in children conceived by in vitro fertilization and intracytoplasmic sperm injection: a meta-analysis". En: Fertility and Sterility. Volume 97, Issue 6, p. 1331-1337.e4, Junio 2012.

Wisburg, K. Ingerslev H.J., Henriksen T.B. "IVF and stillbith: a prospective follow-up study". En: Human Reproduction 2010; 25. 